\title{
S16. Strategies of development in classification
}

\section{THE NEED FOR DEVELOPING NEW CLASSIFICATIONS} IN PSYCHIATRY

\section{P. Pichot}

Académie Nationale de Médicine. 24, rue des Fossés-Saint-Jacques, F75005 Paris, France

Since the publication of the DSM-III, psychiatric classification systems describe, outside of the disorders of proven cause, the categories as clusters of symptoms (diagnostic criteria), the goals being to increase the reliability and to maximise the homogeneity of each category. While the first has been obtained, negative consequences of those principles are evident. Despite the continuous increase of the number of categories, the symptomatic homogeneity of each does not correspond to an intrinsic unity, as demonstrated by the abnormally high level of so-called co-morbidity found in epidemiological studies and by the lack of congruence between diagnostic category and reactivity to a drug having specific pharmacological properties. On the other hand, the hierarchical structure inherent to a nosography relies no more on psychopathological concepts (hence the disappearance of classes such as endogenous psychoses, neuroses and, at another level, of hysteria). The new higher classes, based often on a single symptom (i.e. anxious disorders) lack obviously homogeneity, while formerly regrouped manifestations of hysteria are scattered among widely different classes. Neither from the heuristic nor from the pragmatic point of view the present trend is satisfactory. Among the possible new directions to explore are the use of a dimensional model for all or a part of the mental disorders and/or, in the case of the disorders for which a categorical model appears more satisfactory, the use of the data revealed by epidemiology and by psychopharmacology to define a nosographic hierarchical structure.
PSYCHIATRIC TAXONOMY: SPECIFICATION OR DIMENSIONAL ANALYSIS?

\section{HJ Eysenck}

Institute of Psychiatry, University of London, De Crespigny Park, Denmark Hill, London SE5 BTH, England.

Psychiatry has always followed the medical habits of talking about separate disease entities, like schizophrenia, manic depressive illness etc. There has been much criticism because the reliability of diagnoses based on such a system was always low, and there was quite obviously considerable overlap between different diagnostic units. An alternative system is the dimensional one, in which a person is not diagnosed as suffering from a specific disorder, but is represented by a point in a multidimensional universe where the dimensions are determined by actual experimental and psychometric research.

DSM-3 and later products were designed to improve the situation, but they have resulted in a very much larger number of disease entities, without any great improvement in the actual reliability of such dimensional approach obviates many of the problems, and there is experimental support for such a view. In particular, neurotic and psychotic disorders seem to lie along dimensions of neuroticism and psychoticism, which, together with a third dimension of extraversion/introversion, give a much more realistic picture of the situation, and provide a much better basis for research. 
THE MULTIPLE MEANINGS OF THE EXCESS CO-OCCURRENCE OF PSYCHIATRIC DIAGNOSES

W. Maier, D. Lichtermann, J. Minges, R. Heun

Department of Psychiatry, University of Mainz

Classical psychiatric nosology assumed hierarchical exclusion rules between the various nosological entities in order to avoid multiple diagnostic assignments and so did previous versions or the most frequently used diagnostic manuals. This rationale was (and perhaps still is) widely accepted by clinicians. General population and primary care surveys suggested the boundaries between the most common syndromes (depression, anxiety, substance abuse) to be less distinct with substantial symptomatic overlap. It was also demonstrated that hierarchical rules applied to these conditions ignored relevant differences between subgroups with regard to psychosocial functioning, course and outcome; hierarchy-free diagnostic schedules are considered to provide a more valid rationale for psychiatric classification. The adoption of this principle revealed co-occurrence of different lifetime diagnoses within the same subject to occur substantially more frequently than expected by chance (excess comorbidity) in epidemiological settings.

There may be multiple reasons for excess of co-occurrence of different lifetime diagnoses. Currently, the most powerful tool for exploring excess comorbidity are genetic family studies; these studies are informative if the comorbid disorders are both familial as it is the case for most psychiatric disorders. The following different reasons of excess comorbidity can be teased apart be these tools: a) comorbidity might be due to sharing of familial factors of etiological relevance between both syndromes; b) comorbidity might be due to nonfamilial factors (in particular the presence of one disorder might cause the other one); c) co-occurrence of two lifetime diagnoses in one subject might delineate a distinct nosological entity.

Family studies on dementia and depression and on anxiety disorders, alcohol and drug abuse will be presented to identify sources for the excess co-occurrence of lifetime diagnoses between these disorders. Mainly a) and b) emerge as possible explanations for excess comorbidity. Thus, the empirical results challenge the clam of psychiatric nosology to identify "disease entities".

\section{THE RUBRIC OF BIPOLAR DISORDERS}

IF Brockington

University Department of Psychiatry, Queen Elizabeth Psychiatry Hospital, Mindelsohn Way, Edgbaston, Birmingham, GB

ICD-10 and DSM IV (draft criteria) still base the classification of the psychoses on the distinction between schizophrenia (including schizoaffective, catatonic and persistent delusional disorders) and mood (affective) disorders. It would be better to identify a separate group of bipolar disorders, including manic depression in all its varieties, cycloid psychosis, catatonia and the puerperal/menstrual group, but excluding unipola depression. The recognition of this as an integrated group would clarify the nosology of 'schizophrenia' and depression, and focus research on the aetiology and pathogenesis of the bipolar group.

Recent research into the aetiology of puerperal and menstrual psychosis will be invoked to illustrate the advantage of isolating the bipolar concept from other psychoses.
PSYCHOPHARMACOLOGY FACING CURRENT

DIAGNOSTIC CLASSIFICATIONS LA PSYCHOPHARMACOLOGIE FACE AUX ClasSIFICATIONS DIAGNOSTIQUES ACTUELLES

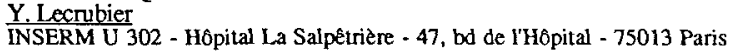

Most reference drugs currently used in therapeutics were discovered in the 1950 's. Since the knowledge on psychotropic action was restricted to clinical efficacy the classification developed in the 1960s was based on the diagnosis of the patients improved. A very broad classification was used (depressed, anxious and psychotics) as the target of antidepressants, anxiolytics and antipsychotics. Although no data show a relationship between the mechanism of action of drugs and the biology of the corresponding disorders, this classification based on the improvement of "a diagnosis" is still mandatory, specially for regulatory agencies. Behavioural pharmacology selected new compounds based on the existence of responses in Animal similar to those observed with reference compounds. As a consequence most new drugs are very similar to the first discovered compounds.

Two other strategies gained acceptance to classify new agents. One is based on their effects on CNS : we now have Specific Serotonin Reuptake Inhibitors (SSRIs), Dopaminergic D2 blockers, Serotonin 5HT1A or 5HT2 agonists or blockers, benzodiazepine BZ1, BZ2 or BZ3 agonists, antagonists or inverse agonists.

Although many new agents show a unique and well identified interaction with CNS their utility in therapeutics is no more restricted to a single nosographic well defined population. SSRIs improve affective, panic and obsessive compulsive disorders. It could be argued that all these disorders are depending from a common superstructure but, at least for some of them, this hypothesis is very unlikely. 5HT1A agonists or 5HT2 blockers obviously improved patients but did not found their "nosological" target. Some dopaminergic blockers proved to be effective in psychotic patients and also in some depressed while "atypical" drugs were shown to be effective in psychotics. All taken together this suggests that most compounds are useful through functional effects on the CNS like, as a comparison, beta-adrenergic blockers are useful in hypertension. Since our knowledge on brain receptors and structures improved exponentially, potentially therapeutic new drugs will be discarted if their clinical efficacy is not described and possibly marketed in terms of "clinical properties" better than in terms of "nosological target". 